OPEN ACCESS

Edited by:

Danielle DeNigris,

Fairleigh Dickinson University,

United States

Reviewed by:

Janie Busby Grant,

University of Canberra, Australia

Wing Chee So,

The Chinese University of Hong Kong,

Hong Kong

*Correspondence:

Petra Hendriks

p.hendriks@rug.nl

Specialty section: This article was submitted to

Cognition,

a section of the journal

Frontiers in Psychology

Received: 18 April 2018

Accepted: 20 August 2018

Published: 05 September 2018

Citation:

Overweg J, Hartman CA and

Hendriks P (2018) Temporarily Out of Order: Temporal Perspective Taking in Language in Children With Autism

Spectrum Disorder.

Front. Psychol. 9:1663.

doi: 10.3389/fpsyg.2018.01663

\section{Temporarily Out of Order: Temporal Perspective Taking in Language in Children With Autism Spectrum Disorder}

\author{
Jessica Overweg ${ }^{1}$, Catharina A. Hartman ${ }^{2}$ and Petra Hendriks ${ }^{1 *}$ \\ ${ }^{1}$ Center for Language and Cognition Groningen (CLCG), University of Groningen, Groningen, Netherlands, ${ }^{2}$ Department \\ of Psychiatry, University Medical Center Groningen, Groningen, Netherlands
}

Clinical reports suggest that children with autism spectrum disorder (ASD) struggle with time perception, but few studies have investigated this. This is the first study to examine these children's understanding of before and after. These temporal conjunctions have been argued to require additional cognitive effort when conjoining two events in a clause order that is incongruent with their order in time. Given the suggested time perception impairment and well-established cognitive deficits of children with ASD, we expected them to have difficulties interpreting temporal conjunctions, especially in an incongruent order. To investigate this, the interpretation of before and after in congruent and incongruent orders was examined in 48 children with ASD and 43 typically developing (TD) children (age 6-12). Additional tasks were administered to measure Theory of Mind (ToM), working memory (WM), cognitive inhibition, cognitive flexibility, IQ, and verbal ability. We found that children with ASD were less accurate in their interpretation of temporal conjunctions than their TD peers. Contrary to our expectations, they did not have particular difficulties in an incongruent order. Furthermore, older children showed better overall performance than younger children. The difference between children with ASD and TD children was explained by WM, ToM, IQ, and verbal ability, but not by cognitive inhibition and flexibility. These cognitive functions are more likely to be impaired in children with ASD than in TD children, which could account for their poorer performance. Thus, the cognitive factors found to affect the interpretation of temporal language in children with ASD are likely to apply in typical development as well. Sufficient WM capacity and verbal ability may help children to process complex sentences conjoined by a temporal conjunction. Additionally, ToM understanding was found to be related to children's interpretation of temporal conjunctions in an incongruent order, indicating that perspective taking is required when events are presented out of order. We conclude from this that perspective-taking abilities are needed for the interpretation of temporal conjunctions, either to shift one's own perspective as a hearer to another point in time, or to shift to the perspective of the speaker to consider the speaker's linguistic choices.

Keywords: autism spectrum disorder, executive functioning, perspective taking, temporal conjunctions, Theory of Mind 


\section{INTRODUCTION}

Time is an important dimension by which we make sense of the world (Navon, 1978). Time is also deeply rooted in the structural organization of language (Klein, 1994). In language, time is generally conceived as a sequential order of events, where one event follows another from past to present to future. Speakers can use temporal expressions, like before or after, to express the order of events in time either in order of occurrence (i.e., temporally congruent) or out of order (i.e., temporally incongruent). The interpretation of the temporal conjunctions before and after in an incongruent order is found to be difficult for typically developing (TD) children (Clark, 1971; Pyykkönen and Järvikivi, 2012; Blything et al., 2015; de Ruiter et al., 2018). This may hold even more for children with an autism spectrum disorder (ASD). Clinical reports suggest that children with ASD encounter difficulties in time perception (Wing, 1996). Additionally, some studies have suggested that individuals with ASD have difficulty interpreting before and after (Boucher, 2001; Perkins et al., 2006). The present study investigates time perception in language in children with ASD and their TD peers by examining their interpretation of sentences containing temporal conjunctions.

Before and after are viewed as the prototypical linguistic expressions indicating temporal order (Schilder and Tenbrink, 2001). Speakers can use these expressions in several ways to express the order of events. For example, all four sentences below indicate that someone first climbed a tree and next read a book:

(1) He climbed the tree before he read the book.

(2) Before he read the book, he climbed the tree.

(3) He read the book after he climbed the tree.

(4) After he climbed the tree, he read the book.

The speaker's choice of before in a main-subordinate clause order (1) and after in a reversed clause order (4) result in a congruent presentation of the temporal order of events, whereas before in a subordinate-main clause order (2) and after in a reversed clause order (3) result in an incongruent presentation. Thus, it depends on the speaker's choice of type of conjunction and clause order whether the hearer should interpret the event order as congruent or incongruent.

Developmental studies in TD children report that congruency has an effect on the correct interpretation of before and after (Clark, 1971; Trosborg, 1982; McCormack and Hanley, 2011; Pyykkönen and Järvikivi, 2012; Blything et al., 2015; de Ruiter et al., 2018). Children under the age of 7 have more difficulties interpreting conjunctions in a temporally incongruent order than in a temporally congruent order, and mostly rely on the order of presentation of the events. Pyykkönen and Järvikivi (2012) showed that children between 8 and 12 years old still experience difficulties interpreting temporal conjunctions in an incongruent order, especially when the cue to event order occurs sentencemedially, as in example sentence (3).

Children's difficulties with interpreting temporal conjunctions in an incongruent order have been explained in various ways. For example, these difficulties have been argued to result from a still fragile understanding of the meaning of the temporal conjunctions before and after (Clark, 1971), from difficulty shifting one's perspective to a different point in time (McCormack and Hoerl, 1999; McCormack and Hanley, 2011), from difficulty processing subordinate-main clause orders (Diessel, 2008), and from difficulty holding information active in working memory (WM) during processing to create a chronological mental representation of the events (Blything et al., 2015; Blything and Cain, 2016). In adults, interpreting temporal conjunctions in an incongruent rather than congruent order comes with processing costs and has been shown to tax WM (Münte et al., 1998). So, interpreting temporal conjunctions in an incongruent order may require additional cognitive effort.

According to anecdotal evidence and clinical reports, individuals with ASD encounter difficulties in time perception (Wing, 1996). They often report a need to adhere to rituals and routines and are commonly preoccupied with timetables, clocks, and calendars, which may serve to compensate for their failure to predict future events and their disorientation in time (Allman and DeLeon, 2009). This led Boucher (2001) to suggest that individuals with ASD have an impaired sense of time. So far, few studies have been conducted on time perception in children with ASD. Some studies report intact time perception (Wallace and Happé, 2008; Gil et al., 2012), while other studies suggest that children with ASD experience particular difficulties with understanding temporal ordering and concepts such as duration, succession, past, and future (Gillberg and Peeters, 1995; Boucher et al., 2007; Maister and Plaisted-Grant, 2011). Also, some studies report that children with ASD use fewer temporal expressions in story-telling (Colle et al., 2008) and more often omit tense marking than their TD peers (Roberts et al., 2004). These findings regarding the production of temporal expressions suggest that children with ASD may struggle with their interpretation of temporal conjunctions as well, although a mismatch between their production abilities and their comprehension abilities is also conceivable (see Hendriks, 2014 for an overview and discussion of attested production-comprehension asymmetries in child language).

Executive functioning (EF) impairments, often present in children with ASD (Hill, 2004), could make it especially difficult to interpret temporal conjunctions in an incongruent order. EF refers to cognitive processes such as WM (the capacity system that allows the temporary storage and manipulation of information necessary for complex tasks such as language comprehension; Baddeley, 2000), inhibition (the mental ability to suppress irrelevant information; Dagenbach and Carr, 1994), and flexibility (the mental ability to shift between different thoughts or actions; Scott, 1962), that allow for the flexible alteration of thought and behavior in response to changing contexts (Welsh and Pennington, 1988). Recent studies have argued that TD children between 3 and 7 years old have more difficulties interpreting temporal conjunctions in an incongruent order than in a congruent order because more information must be maintained in WM to revise the mental representation of the events and create a chronological mental representation (Blything et al., 2015; Blything and Cain, 2016). The neuroimaging studies of Münte et al. (1998) and Ye et al. (2012) suggest that, also for adults, WM is needed for the temporal re-ordering of events. Furthermore, the ability to 
inhibit an initial interpretation and to flexibly revise a mental representation of event order could be needed to interpret conjunctions in an incongruent order (Pyykkönen and Järvikivi, 2012; Blything and Cain, 2016). Thus, in addition to WM, also cognitive inhibition and cognitive flexibility may be involved.

In addition to impairments in these EF functions, also impairments in Theory of Mind (ToM) understanding (Frith and Frith, 2006) could make it difficult for children with ASD to interpret temporal conjunctions in an incongruent order. ToM is the ability to take the cognitive perspective of other people to understand their beliefs, desires and intentions (Wimmer and Perner, 1983) and is argued to be impaired in children with ASD (Baron-Cohen et al., 1985). If the interpretation of an incongruent temporal order involves ToM understanding, an incongruent temporal order may be especially difficult for children with ASD. Several studies have suggested that the interpretation of temporal language not only requires a consideration of the actual perspective in time but also a consideration of alternative temporal perspectives (McGlone and Harding, 1998; McCormack and Hoerl, 1999; Stocker, 2012). According to McCormack and Hoerl (1999), hearers should not only be able to shift from the actual perspective in time to alternative temporal perspectives, but should also understand the relation between these perspectives. Based on their account of the development of temporal understanding, they posit that "temporal perspective taking involves mentalizing abilities" (McCormack and Hoerl, 1999; p. 174). Thus, mentalizing, or ToM understanding, could be involved in the comprehension of an incongruent order of events.

This is the first study to investigate how 6- to 12-yearold children with ASD and their TD peers interpret temporal conjunctions. We expect that all children find the interpretation of before and after more difficult in the incongruent order than in the congruent order, but that children with ASD find the interpretation of these temporal conjunctions in an incongruent order more difficult than their TD peers. As EF and ToM have been reported to be possibly impaired in individuals with ASD, this may explain the hypothesized difficulties with the interpretation of temporal conjunctions in children with ASD. Therefore, we further hypothesize that differences in the interpretation of temporal conjunctions in an incongruent order are associated with individual differences in EF and ToM understanding. In addition to the specific cognitive factors EF and ToM, we also examine the role of the more general cognitive factors IQ and verbal ability. EF and ToM may not only provide insight into the individual differences in ASD that play a role in temporal language understanding, but may also provide insight into what it is in the broad measures of IQ and verbal ability that possibly explains temporal language understanding.

\section{MATERIALS AND METHODS}

\section{Participants}

In this study, 48 children with ASD and 43 TD children participated. All children were monolingual native Dutch children who did not have any reported language disorders.
The children in the ASD group were diagnosed with ASD by clinicians on the basis of the DSM-IV-TR criteria (American Psychiatric Association, 2000) and had an IQ of $>75$ based on a clinically administered full IQ test. Additionally, in all children (ASD as well as TD), certified professionals administered the Autism Diagnostic Observation Schedule (ADOS; Lord et al., 1999), the Autism Diagnostic Interview Revised (ADI-R; Rutter et al., 2003), two subtests (Vocabulary and Block Design) of the WISC-III-NL to estimate IQ (Kort et al., 2002), and the Peabody Picture Vocabulary Test to measure Verbal Ability (VA) (PPVTIII-NL; Schlichting, 2005). Two children from the ASD group were excluded because they neither met the ADOS criteria for ASD nor the ADI-R criteria for ASD (cf. Risi et al.'s., 2006, ASD2 criteria). One child from the TD group met the ADOS criteria for ASD and was therefore excluded as well, leaving 46 children with ASD (mean age $=9 ; 4, \mathrm{SD}=2 ; 2$ ) and 42 TD children (mean age $=9 ; 2, S D=2 ; 0)$ for further analysis. The group descriptives of the ASD group and the TD group are provided in Table $\mathbf{1}$.

Children with ASD were recruited via outpatient clinics for child and adolescent psychiatry in Groningen and a national website for parents with children with ASD. TD children were recruited via advertising in newsletters and flyers at schools in the north of Netherlands. The children were tested individually on a single day in a quiet room at the university with two experimenters present. This study is part of a wider study on language and perspective taking in children with ASD, in which all children of the current study participated. The medical ethical committee of the University Medical Hospital Groningen evaluated this study as not falling under the Medical Research Involving Human Subjects Act (WMO). Nevertheless, we followed the required procedures and obtained written informed consent from the parents of all participants for their child's participation in the research.

\section{Language Comprehension Task}

Comprehension of temporal conjunctions was tested using a picture selection task. Per item, participants saw two pictures side by side on a computer screen, each depicting an event (see Figure 1).

Simultaneously, they heard a pre-recorded sentence describing the temporal order of the two events. Participants had to press one of two buttons on a button box to select the picture that, according to the sentence, showed the event that happened first. The sentences contained either voordat ("before") or nadat ("after"), which occurred either in sentenceinitial position (corresponding to subordinate-main clause order) or in sentence-medial position (corresponding to main-subordinate clause order). Examples of each of the four conditions (conjunction $\times$ position) in the language comprehension task are shown below in Dutch, followed by word by word glosses and English translations:

(1) voordat ("before") in sentence-initial position: Voordat hij het boek las, klom hij in de boom. before he the book read, climbed he in the tree "Before he read the book, he climbed the tree."

(2) voordat ("before") in sentence-medial position: 
TABLE 1 | Description of the participants with autism spectrum disorder (ASD) and the typically developing (TD) participants in this study.

\begin{tabular}{|c|c|c|c|}
\hline $\begin{array}{l}\text { Background } \\
\text { variables }\end{array}$ & ASD $(N=46)$ & $\mathrm{TD}(N=42)$ & $\begin{array}{c}\text { Group differences } \\
\text { (general linear } \\
\text { model ANOVA } \\
\text { analyses) }\end{array}$ \\
\hline Gender (boys:girls) & $39: 7$ & $34: 8$ & n.s. \\
\hline \multicolumn{4}{|l|}{$\begin{array}{l}\text { Chronological age } \\
\text { (year; month) }\end{array}$} \\
\hline Mean (SD) & $9 ; 4(2 ; 2)$ & $9 ; 2(2 ; 0)$ & n.s. \\
\hline Range & $6 ; 0-12 ; 5$ & $6 ; 2-12 ; 7$ & \\
\hline \multicolumn{4}{|l|}{$\begin{array}{l}\text { Clinical diagnosis } \\
\text { of ASD subtype } \\
\text { according to } \\
\text { DSM-IV criteria }(N)\end{array}$} \\
\hline Autistic disorder & 4 & 0 & - \\
\hline Asperger's disorder & 2 & 0 & - \\
\hline PDD-NOSa & 40 & 0 & - \\
\hline \multicolumn{4}{|l|}{$\begin{array}{l}\text { Number of } \\
\text { participants } \\
\text { meeting ASD2 } \\
\text { criteriab on }^{\text {b }}\end{array}$} \\
\hline ADOS and ADI & 33 & 0 & - \\
\hline ADOS only & 10 & 1 (excluded) $^{e}$ & - \\
\hline ADI only & 3 & 0 & - \\
\hline $\begin{array}{l}\text { Neither ADOS nor } \\
\text { ADI }\end{array}$ & 2 (excluded) $^{e}$ & 42 & - \\
\hline \multicolumn{4}{|l|}{$\begin{array}{l}\text { Estimated IQ } \\
\text { (WISC) }^{c}\end{array}$} \\
\hline Mean $(S D)$ & 99.87 (16.92) & $113.21(13.86)$ & $\mathrm{TD}>\mathrm{ASD}^{* * *}$ \\
\hline Range & $66.65-145.48$ & $72.71-145.48$ & \\
\hline \multicolumn{4}{|l|}{$\begin{array}{l}\text { Verbal ability score } \\
\text { (PPVT) }^{d}\end{array}$} \\
\hline Mean $(S D)$ & $104.48(13.9)$ & 113.62 (11.53) & $\mathrm{TD}>\mathrm{ASD}^{* *}$ \\
\hline Range & $77-139$ & $87-138$ & \\
\hline
\end{tabular}

a PDD-NOS: pervasive developmental disorder-not otherwise specified; ${ }^{b}$ The ASD2 criteria of Risi et al. (2006) are: "a child meets criteria on Social and Communication domains or meets criteria on Social and within two points of Communication criteria or meets criteria on Communication and within two points of Social criteria or within one point on both Social and Communication domains" (Risi et al., 2006; p. 1100); ${ }^{c}$ Estimated IQ of two subtests of the Dutch version of the Wechsler Intelligence Scale for Children (WISC-III-NL; Kort et al., 2002); ' Normed verbal ability score from the Dutch version of the Peabody Picture Vocabulary Test (PPVT-III-NL; e Excluded from the group descriptives in this table as well as from the analyses; Schlichting, 2005); ${ }^{* *} p<0.01 ;{ }^{* * *} p<0.001$.

Hij klom in de boom voordat hij het boek las. he climbed in the tree before he the book read "He climbed the tree before he read the book."

(3) nadat ("after") in sentence-initial position: Nadat hij in de boom klom, las hij het boek. after he in the tree climbed, read he the book "After he climbed the tree, he read the book."

(4) nadat ("after") in sentence-medial position: Hij las het boek nadat hij in de boom klom. he read the book after he in the tree climbed "He read the book after he climbed the tree."

The events in sentences (2) and (3) are mentioned in a congruent order, whereas the events in (1) and (4) are mentioned in an incongruent order. All events were unrelated to avoid a preference for one of the two event orders based on event typicality.

Stimuli were presented and responses were recorded using the computer software E-Prime 2.0 (Schneider et al., 2002). First, children completed three practice items to practice that the left and right button corresponded to the left and right picture, respectively. This was followed by an introduction of the boy in the pictures and three practice items containing other temporal expressions (e.g., "today" and "yesterday") to determine whether the participant understood the principle of temporal ordering in the task. Next, the participants received 32 test items, with a short break in the middle. The test items were distributed across 4 lists. Each list contained 16 congruent test items and 16 incongruent test items in a randomized order. We counterbalanced the position of the pictures on the screen. The experiment took approximately $15 \mathrm{~min}$.

\section{Cognitive Tasks Working Memory}

To test WM, the N-Back task (Owen et al., 2005) was used. In this task, participants had to watch and remember pictures presented one by one on a computer screen and indicate whether the picture on the screen was a particular object or not (0-back or baseline condition), whether it matched the picture one trial before (one-back condition), and whether it matched the picture two trials before (two-back condition). Participants received a practice session of 15 trials per condition and a test session consisting of 60 trials per condition. The mean accuracy (ACC) on the two-back condition was calculated as a measure of WM.

\section{Cognitive Inhibition}

To test cognitive inhibition, the Flanker task [Amsterdam Neuropsychological Test battery (ANT) version 2.1; De Sonneville, 1999] was administered. In this task, participants had to identify the color of a target stimulus surrounded by eight distractors (flankers). The target color was red or green and was associated with the left or right button, respectively. The flankers were either in the same color as the target (compatible trials) or in the color that was associated with the opposite response (incompatible trials). For this task, participants received 12 practice items, 40 compatible test items, and 40 incompatible test items. The mean ACC and mean reaction time (RT) of cognitive inhibition was measured by subtracting the mean ACC or RT on compatible trials from the mean ACC or RT, respectively, on incompatible trials (resulting in the congruency effect; see Mullane et al., 2009).

\section{Cognitive Flexibility}

To test cognitive flexibility, we adapted the gender emotion switch task of De Vries and Geurts (2012) to make it more similar to a classical switch task (e.g., Rogers and Monsell, 1995). In our shape-color switch task, participants saw pictures of round or square figures in black or white on the computer screen and had to press the left or right button to report the shape (round or square) or the color (black or white) of the figure. The cue at the top of the screen indicated whether the shape or the color had to 

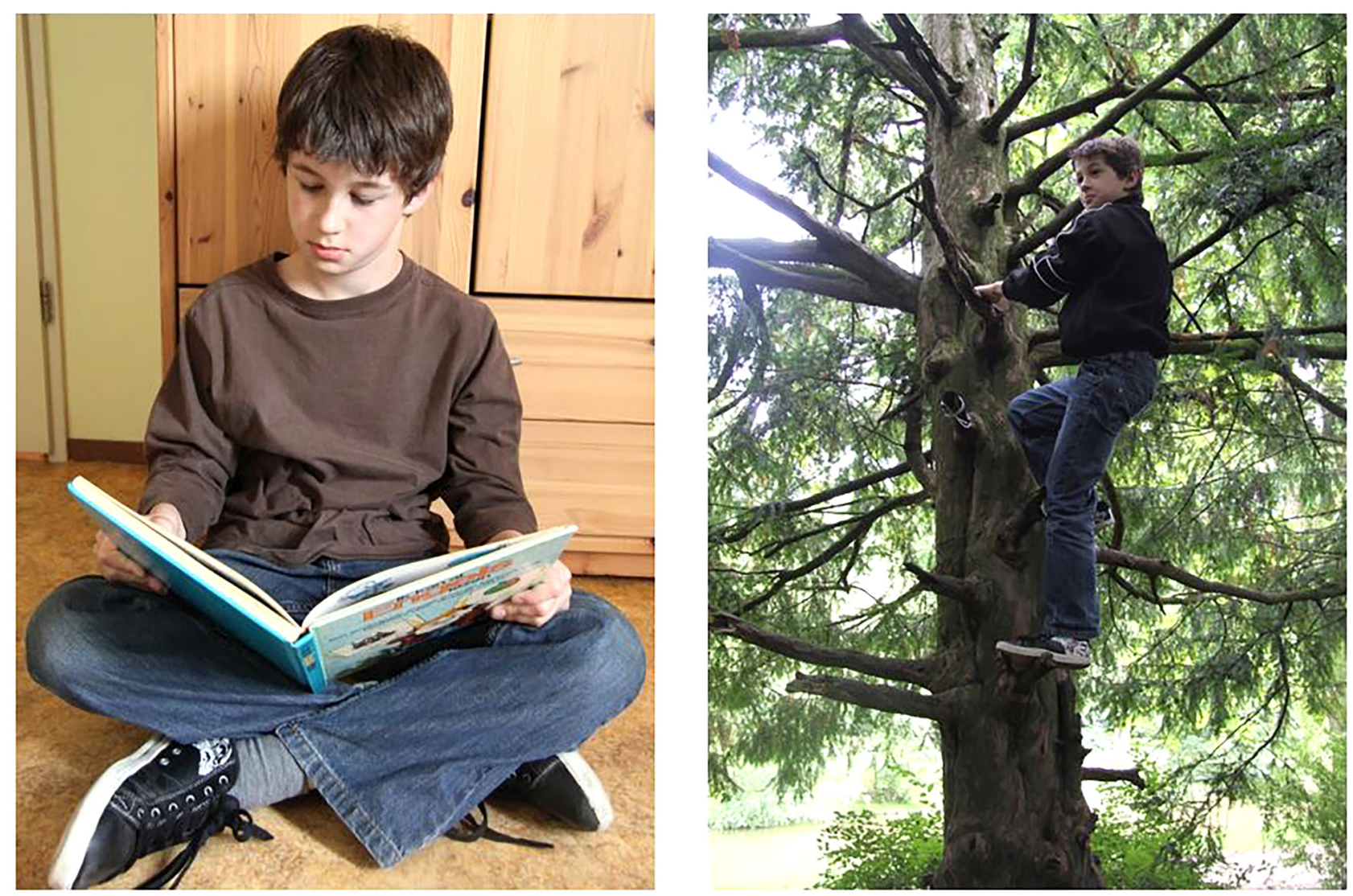

FIGURE 1 | An example of the two pictures of an item in the language comprehension task. Written informed consent was obtained from the parents for publication of their child's images.

be reported. Participants received 16 items to practice with shape, 16 items to practice with color, and 40 items to practice with switching between shape and color. The test consisted of 216 trials in total; a third of these trials (72) were switch trials (switching from color to shape or vice versa) and the remaining two third were repeat trials. The mean ACC and mean RT of switch costs was measured by subtracting the mean ACC or RT on repeat trials from the mean ACC or RT, respectively, on switch trials (cf. De Vries and Geurts, 2012).

\section{Theory of Mind}

To test first-order and second-order ToM, the Bake Sale task adapted from Hollebrandse et al. (2014) was used. This task is a second-order false belief (FB) task with stories modeled after Perner and Wimmer's (1985) “ice cream truck story" in which the beliefs of various characters were manipulated. Per story, participants heard a verbal description of the events in the story, accompanied by four pictures that were presented one by one. During the presentation of the story, they received three questions to probe their understanding of the events in the story, as well as a question about the FB of another person (first-order $\mathrm{FB}$ question) and a question about the $\mathrm{FB}$ of another person about a second person (second-order FB question). The task consisted of eight stories in total, each of which contained a first-order
FB question and a second-order FB question. The measures of ToM1 and ToM2 were calculated using the ACC on the eight first-order FB questions and the ACC on the eight second-order FB questions, respectively.

\section{Data Analysis}

The data of the language comprehension task were analyzed using generalized linear mixed models (GLMMs), using a logit link to accommodate the repeatedly measured (32 trials) binary outcome variable Accuracy (0 for incorrect, 1 for correct) (Jaeger, 2008; Heck et al., 2012). Compound symmetry was used as the covariance matrix type. We set out with a full factorial model with Congruency (Congruent vs. Incongruent) as within group factor and Group (TD vs. ASD) as between group factor. Age was meancentered and additionally included in the model. Interactions that did not have an effect on Accuracy $(p>0.05)$ were removed from the model one by one, starting with the interaction with the largest $p$-value, after which we refitted the model. This resulted in model 1, which shows the extent to which Accuracy was predicted by Congruency, Group, and Age, as well as the relevant $(p<0.05)$ interactions. The possible presence of effects related to Type of conjunction (Before vs. After) and Clause order (Mainsubordinate vs. Subordinate-main) were subsequently checked, 
post hoc, in model 1. For purposes of interpretation, we illustrate significant effects using the median split method.

Next, the seven parameters derived from the N-Back task (WM), the Flanker task (Cognitive inhibition ACC and Cognitive inhibition RT), the cognitive flexibility task (Switch costs ACC and Switch costs RT) and the FB task (ToM1 and ToM2) were mean-centered and, one by one, examined as main effects and in interaction with the significant predictors from model 1 in seven separate analyses. The data of 3 participants (2 ASD and 1 TD) were missing in the Cognitive inhibition ACC and RT analyses, leaving the data of 44 participants with ASD and 41 TD participants. In each separate analysis, interactions that had no effect on Accuracy $(p>0.05)$ were removed from the model. Based on the outcomes of these analyses per predictor, we combined the cognitive factors with (main or interaction) effects on Accuracy $(p<0.05)$ and added these with the significant predictors of model 1 in a model with multiple predictors to evaluate their effects adjusted for one another (cf. Kuijper et al., 2015; Overweg et al., 2018). This resulted in model 2, which shows the relevant cognitive factors that had an effect on the interpretation of temporal conjunctions.

Finally, the parameters from the WISC (estimated IQ on the basis of the subtests Vocabulary and Block Design) and PPVT (VA) were mean-centered and included in two separate analyses in model 1. If they had an effect on Accuracy $(p<0.05)$, they were added to model 2 and evaluated in model 3. This resulted in model 3, which shows whether these general background variables changed the effects found in model 2. Given the significant group differences (see Table 1) in estimated IQ and VA, this approach provides a statistical alternative to a priori matching on estimated IQ and VA.

\section{RESULTS}

Model 1 showed main effects of Group and Age, indicating that the children in the TD group were more accurate in their interpretation of temporal conjunctions than the children in the ASD group, and that the older the child was, the better its performance. No main effect or interactions with Congruency were found (all $p$-values $>0.05$ ). A post hoc exploration of Type of conjunction and Clause order in model 1 showed a main effect of Type of conjunction $(B=-0.943 ; S E=0.14 ; p=0.00)$, indicating that children perform better on sentences with before than on sentences with after. Clause order did not influence performance $(p>0.05)$. Table 2 lists all remaining effects in model 1 .

Figure 2 presents the mean proportions of correct responses in the congruent and incongruent condition separately for the ASD and TD groups.

Next, we examined one by one which cognitive factors were associated with Accuracy. The separate analyses indicated a main effect of WM $(B=2.355 ; S E=0.747 ; p=0.002)$ and interactions of ToM $1^{*}$ Congruency $(B=2.325 ; S E=1.034 ; p=0.026)$ and ToM2*Congruency $(B=1.465 ; S E=0.552 ; p=0.009)$. No effects of Cognitive inhibition and Cognitive flexibility were found $(p-$ values $<0.05$ ).
Then, we combined all significant interactions and main effects of these analyses per predictor in model 2, a model with multiple predictors. The interaction effect of ToM1*Congruency was no longer significant when adjusted for the other cognitive variables and was removed from the model. Table 2 lists all remaining effects in model 2.

Model 2 showed a main effect of WM ( $p=0.03$; see Table 2), indicating that children with lower WM are less accurate in their interpretation of temporal conjunctions than children with higher WM. Model 2 also showed an interaction effect of ToM2*Congruency ( $p=0.01$; see Table 2 ). As is shown in Figure 3, children with lower second-order ToM understanding are less accurate in their interpretation of temporal conjunctions in the Incongruent condition than children with higher secondorder ToM understanding. The median split method is used to plot Accuracy of temporal conjunction interpretation in each condition per ToM2 group (low ToM2: $\leq 0.75$ vs. high ToM2: $>0.75)$ to illustrate the direction of the interaction effect. The figure caption of Figure 3 provides background information about the ToM performance of each group.

The main effects of Group and Age disappeared with the addition of ToM 2 and WM in model 2 (all $p$-values $>0.05$; see Table 2).

Finally, we checked for possible effects of the background variables IQ and VA on Accuracy. These analyses per predictor indicated main effects of IQ $(B=0.026 ; S E=0.005 ; p<0.001)$ and VA $(B=0.033 ; S E=0.006 ; p<0.001)$ and an interaction effect of VA*Age $(B=0.001 ; S E=0.00 ; p<0.001)$. In model 3 , we combined these main and interaction effects with the effects of model 2. Model 3 showed main effects of IQ and VA, indicating that children with a lower IQ and lower VA show a lower Accuracy than children with a higher IQ and higher VA, respectively. The interaction of $\mathrm{VA}^{*}$ Age remained significant in this analysis with multiple predictors, indicating that younger children (regardless of their VA), and older children with low VA, were less accurate in their interpretation of temporal conjunctions than older children with high VA, as is shown in Figure 4. Again, the median split method is used to plot Accuracy of temporal conjunction interpretation in each condition per VA group to illustrate the direction of the interaction effect. The figure caption of Figure 4 provides background information about the VA performance of each group.

With the addition of IQ and VA, the main effect of WM disappeared $(p>0.05)$. The interaction effect of ToM2*Congruency remained significant in model 3. Together, the results show that second-order ToM, WM, IQ, and VA play a role in the interpretation of temporal conjunctions. Individual and group differences therein explain why the TD group performs better than the ASD group and why older children perform better than younger children.

\section{DISCUSSION}

We investigated time perception in language by examining the interpretation of sentences containing the temporal conjunctions before and after by native Dutch school-aged children with 
TABLE 2 | Estimated effects of variables per model on the interpretation of temporal conjunctions.

\begin{tabular}{|c|c|c|c|c|c|c|c|c|c|}
\hline \multirow[t]{3}{*}{ Variables } & \multicolumn{9}{|c|}{ Models } \\
\hline & \multicolumn{3}{|c|}{ Model 1} & \multicolumn{3}{|c|}{ Model 2} & \multicolumn{3}{|c|}{ Model 3} \\
\hline & Estimate & SE & $p$ & Estimate & SE & $p$ & Estimate & SE & $p$ \\
\hline Intercept & 1.873 & 0.194 & $0.00^{* *}$ & 1.605 & 0.192 & $0.00^{* *}$ & 1.608 & 0.195 & $0.00^{* *}$ \\
\hline Group & -0.549 & 0.191 & $0.01^{*}$ & -0.114 & 0.215 & 0.60 & 0.085 & 0.231 & 0.71 \\
\hline Age & 0.025 & 0.004 & $0.00 * *$ & 0.006 & 0.006 & 0.25 & 0.017 & 0.006 & $0.00 * *$ \\
\hline Congruency & -0.292 & 0.188 & 0.12 & -0.173 & 0.171 & 0.32 & -0.169 & 0.174 & 0.33 \\
\hline ToM1 & - & - & - & 1.253 & 0.813 & 0.13 & 1.051 & 0.744 & 0.17 \\
\hline ToM2 & - & - & - & -0.334 & 0.401 & 0.41 & -0.698 & 0.420 & 0.10 \\
\hline WM & - & - & - & 1.735 & 0.788 & $0.03^{*}$ & 1.204 & 0.722 & 0.10 \\
\hline ToM2Congruency & - & - & - & 1.502 & 0.567 & $0.01^{*}$ & 1.544 & 0.586 & $0.01^{*}$ \\
\hline $\mathrm{IQ}$ & - & - & - & - & - & - & 0.015 & 0.006 & $0.02^{*}$ \\
\hline VA & - & - & - & - & - & - & 0.016 & 0.007 & $0.03^{*}$ \\
\hline $\mathrm{VA}^{\star} \mathrm{Age}$ & - & - & - & - & - & - & 0.001 & 0.000 & $0.00^{* *}$ \\
\hline
\end{tabular}

The models were built with accuracy in the language comprehension task as the dependent variable and the variables listed in the first column as independent variables. The variable Congruency was manipulated by Type of conjunction (Before vs. After) and Clause order (Main-subordinate vs. Subordinate-main), with Before+Mainsubordinate and After+Subordinate-main resulting in Congruent items, and Before+Subordinate-main and After+Main-subordinate resulting in Incongruent items. A post hoc exploration of Type of conjunction and Clause order in model 1 showed a main effect of Type of conjunction ( $B=-0.943 ; S E=0.14 ; p=0.00) ;{ }^{*} p=<0.05$; ${ }^{* *} p=<0.01$

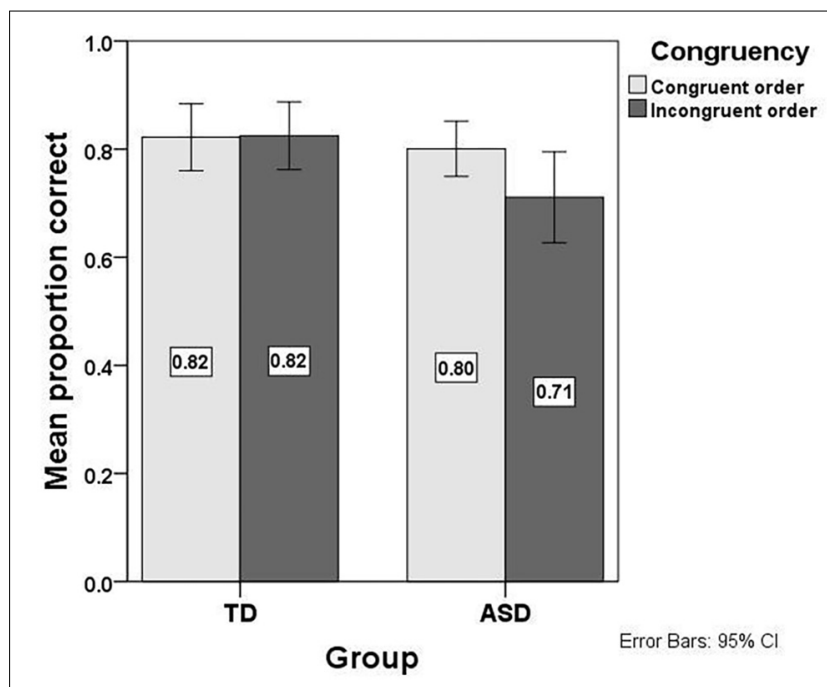

FIGURE 2 | Mean proportion of correct responses in the language comprehension task per Congruency condition (Congruent vs. Incongruent) and Group (TD vs. ASD).

and without ASD. We found, in line with our predictions, that children with ASD were less accurate than their TD peers at interpreting these temporal conjunctions. Contrary to our predictions, however, children with ASD did not have particular difficulties with temporal conjunctions in an incongruent compared to a congruent order. Furthermore, older children were found to perform better than younger children.

To understand the group and age effects, we examined which cognitive factors were associated with the interpretation of temporal conjunctions. Also, we examined whether the general background variables IQ and Verbal Ability affected

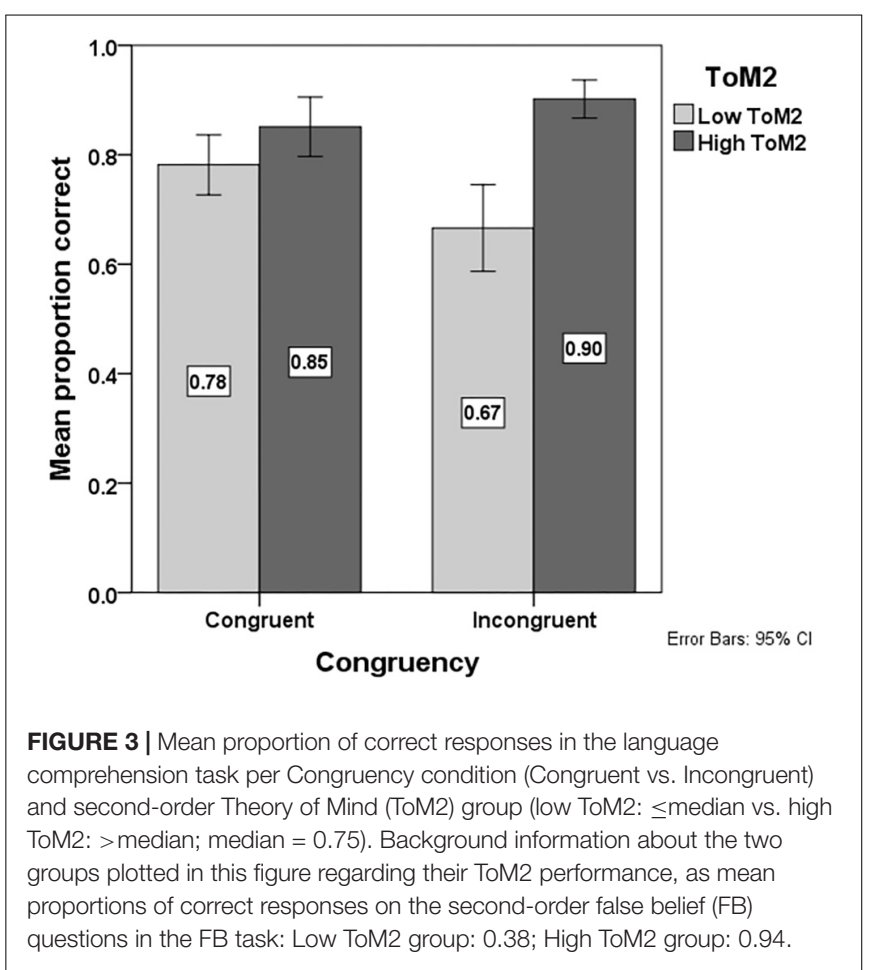

interpretation. We found that age, IQ and VA were the major predictors of children's correct interpretation of temporal conjunctions. Furthermore, the group effect was explained by differences in WM, second-order ToM understanding, IQ and VA. Children with ASD as well as TD children with lower WM made more errors when interpreting temporal conjunctions than children with higher WM. However, the effect of WM disappeared when taking into account children's 


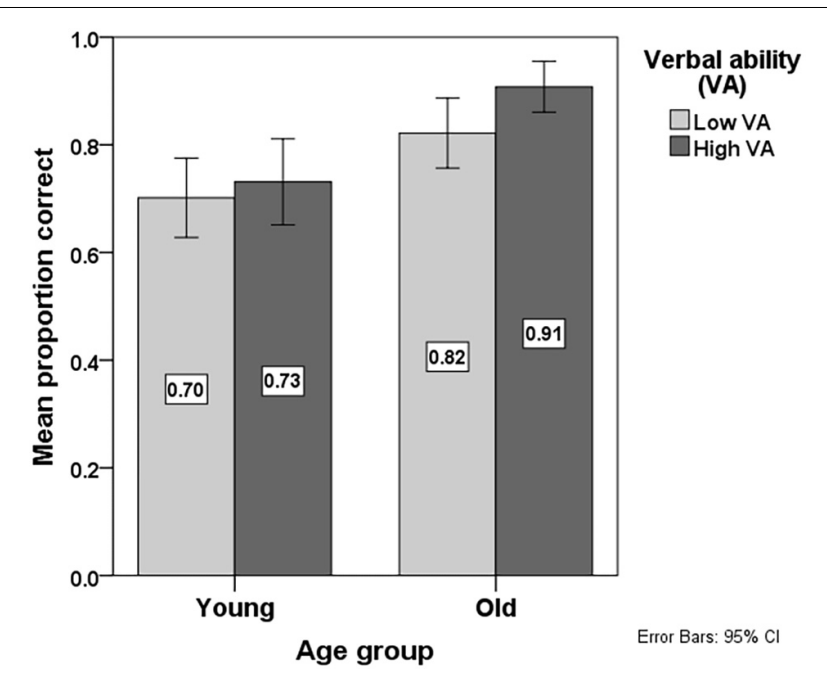

FIGURE 4 | Mean proportion of correct responses in the language comprehension task per Age group (Young: $\leq$ median vs. Old: > median; median = 9;3 years) and Verbal ability (VA) group (low VA: $\leq$ median vs. High VA: $>$ median; median =108.50). Background information about the four groups plotted in this figure regarding their VA, as mean scores in the PPVT: Young-Low VA group: 99.86; Young-High VA group: 121.13; Old-Low VA group: 95.96; Old-High VA group: 118.48 .

IQ. This is not surprising, given the strong relation between WM and IQ (Ackerman et al., 2005; Kidd, 2013). Also, IQ is a more broadly defined cognitive variable than WM and, in addition to measuring the simple short-term storage component of WM (Colom et al., 2008), also measures other cognitive abilities. VA appeared to underlie the age improvement in our study. Younger children, and older children with lower VA, made more errors when interpreting temporal conjunctions than older children with higher VA. This suggests that verbal skills must be sufficiently well developed for a mature understanding of complex sentences such as those involving temporal conjunctions. While suggested by previous studies (McCormack and Hanley, 2011; Blything et al., 2015), we found no effects of cognitive flexibility and cognitive inhibition (cf. de Ruiter et al., 2018). Particularly relevant for our research question and hypotheses was our finding that better secondorder ToM understanding was positively associated with correct interpretation in an incongruent temporal order.

Although most children in our study showed a robust understanding of sentences containing temporal conjunctions, as predicted the children with ASD were less accurate than their TD peers at interpreting these sentences. In line with our hypotheses, this group difference between children with ASD and TD children was explained by differences in WM, secondorder ToM understanding, IQ and VA. Because these cognitive functions are more likely to be impaired in children with ASD than in TD children (see Section "Introduction"), we attribute the poorer performance of children with ASD to their impaired cognitive functions rather than to their clinical diagnosis of ASD per se. Thus, our results actually suggest a much broader application than ASD, as the observed effects of cognitive factors on the interpretation of temporal language are likely to be relevant for typical development as well.

We did not find confirmation for our prediction that children with ASD have particular difficulties with temporal conjunctions in an incongruent order. Also, we did not find a main effect of congruency. The children in our study performed equally well on congruent as on incongruent items, in contrast to what has been found in several earlier studies with TD children (Clark, 1971; Trosborg, 1982; McCormack and Hanley, 2011; Pyykkönen and Järvikivi, 2012; Blything et al., 2015; de Ruiter et al., 2018). Possibly, we did not find a main effect of congruency because the children in our study were on average older (with a mean age of 9) than the children in most earlier studies and can be expected to have a more robust understanding of the meaning of the temporal conjunctions. Only one effect of congruency emerged from our data: children who make more errors in their interpretation of temporal conjunctions in an incongruent order were found to have a lower second-order ToM understanding. Good ToM understanding may thus help children to correctly interpret temporal conjunctions in an incongruent order, thereby suggesting that perspective taking is needed to interpret temporal conjunctions when the events are presented out of order.

One way to explain the role of ToM is that ToM understanding helps children to shift their perspective to another point in time in response to temporal language, and to understand the relationship between these different temporal perspectives on the same events (cf. McCormack and Hoerl, 1999; McCormack and Hanley, 2011). This explanation is in line with the literature on episodic memory based on the notion of mental time travel (Suddendorf and Corballis, 2007), or mental selfprojection (Kretschmer-Trendowicz et al., 2016). Mental time travel involves a shift of the self from the immediate present to an alternative temporal perspective, for example, a past or future perspective (Buckner and Carroll, 2007; Suddendorf and Corballis, 2007). Several studies have suggested that there is a relation between mental time travel abilities and the comprehension of temporal language (Suddendorf and Corballis, 2007; Ferretti and Cosentino, 2013). In addition, it has been found that the neural processes involved in false-belief inferencing and the neural processes involved in mental time travel, in particular in taking the perspective of one's future self to choose between an immediate and a future reward, overlap (O'Connell et al., 2018). In line with our results, this suggests that the comprehension of temporal language involves ToM understanding to enable hearers to shift from the immediate present to another point in time and perceive the situation from these different temporal perspectives.

An alternative possibility is that ToM understanding enables hearers to shift from their own perspective to the perspective of the speaker, for example, to find out why the speaker presented the events in an incongruent order. de Ruiter et al. (2018) explain their finding that children perform better with a congruent than an incongruent order in terms of the semantic principle of iconicity. They suggest that children initially assume an iconic (i.e., congruent) mapping between the order of events in the sentence and the order of events in the real world. Iconicity has been argued elsewhere to result from perspective taking; more 
complex, marked, forms tend to express more complex, marked, meanings (e.g., Horn, 1984; Levinson, 2000; Aissen, 2003). These more complex meanings have been argued to be acquired later in typical development than their less complex counterparts because they require the hearer to reason about why the speaker did not use the less complex form (e.g., De Hoop and Krämer, 2006; Hendriks et al., 2010). Incongruent meanings are more complex than congruent meanings. Also, sentences with after seem to be more complex than sentence with before, considering the post hoc effect of type of conjunction but not of clause order in our study (see note of Table 2) and the observation that before is acquired earlier than after (see Clark, 1971). Thus, a sentence with after may require the hearer to reason about why the speaker chose to use after rather than before, for example to foreground or background particular information. As mentioned above, our results indicate that children who make more errors in their interpretation of temporal conjunctions in an incongruent order have a lower second-order ToM understanding. Good ToM understanding may thus help hearers to correctly interpret temporal conjunctions in an incongruent order by allowing them to take the speaker's perspective to find out why the speaker presented the events out of order.

In contrast to the study of de Ruiter et al. (2018), our study suggests that children need sufficient WM capacity for the interpretation of sentences containing temporal conjunctions. The different findings of the role of WM capacity could be the result of different WM measures. While we used a visuospatial WM task (an N-Back task) to operationalize WM capacity, de Ruiter and colleagues used three short-term memory tasks that do not require manipulation of the stored information (a word repetition task, a non-word repetition task and a sentence imitation task). These tasks may not have captured WM to the extent needed in complex sentence comprehension. Our findings confirm the results of Blything and Cain (2016), who used a verbal WM task (a digit span task) and also found a main effect of WM capacity on the interpretation of temporal conjunctions. Importantly, like Blything and Cain, we did not find that congruency interacted with WM in the accuracy task. This suggests that children's difficulties with interpreting temporal conjunctions in an incongruent order are not explained by insufficient WM. Rather, children seem to need sufficient WM to process complex sentences conjoined by a temporal conjunction in general. These findings are corroborated by studies that have shown that individuals need WM capacity for the comprehension of other types of complex sentences as well, such as relative clauses and complement clauses (Just and Carpenter, 1992; Lewis et al., 2006; Montgomery et al., 2008; Boyle et al., 2013).

Turning to the implications of our study for ASD, previous research on temporal language in children with ASD mostly focused on production, showing deficits in the use of temporal adverbials and tense marking (Roberts et al., 2004; Colle et al., 2008). Here, we showed that verbal children with ASD also struggle with the interpretation of temporal conjunctions, due to weaker ToM understanding and lower WM capacity. This finding highlights the need to further study the interpretation of temporal expressions and temporal ordering in individuals with ASD. Languages have various ways to mark present, past and future and do so in almost every sentence. For example, English has tense marking on the finite verb, temporal adverbials such as now, yesterday, and tomorrow, and in addition to before and after also has other temporal conjunctions such as when, while, and then. A possibility for future research is to examine the interpretation of these and other temporal expressions in children with ASD. A second implication of our study for ASD concerns the nature of the language and communication difficulties in children with ASD. Linguistic deficits in verbal children with ASD are mostly viewed as difficulties with pragmatic aspects of language, which depend on its usage in context (American Psychiatric Association, 2013). However, the interpretation of temporal conjunctions depends on the meaning of the conjunction and its position in the sentence independently of their usage in context, and therefore, difficulty with their interpretation is structural (i.e., syntactic and semantic) rather than pragmatic in nature. In line with previous studies (Boucher, 2012; Durrleman et al., 2015), our results indicate the need to investigate the linguistic deficits in verbal children with ASD beyond pragmatics.

Summarizing, our study showed that children with ASD were less accurate at interpreting sentences containing temporal conjunctions than their TD peers, but did not have more difficulty in an incongruent rather than a congruent order. The different overall performance of children with ASD and TD children was explained by differences in second-order ToM understanding, WM, IQ, and VA, indicating that these factors likely contribute to the mature interpretation of temporal conjunctions. Specifically, second-order ToM understanding was associated with the interpretation of temporal conjunctions in an incongruent order, suggesting that perspective taking is needed to either shift one's own perspective as a hearer from the immediate present to another point in time and relate these different temporal perspectives on the same events, or to shift to the perspective of the speaker to consider the speaker's linguistic choices.

\section{AUTHOR CONTRIBUTIONS}

$\mathrm{JO}, \mathrm{CH}$, and $\mathrm{PH}$ contributed to the conception and design of the study. JO carried out the experiments. JO, $\mathrm{CH}$, and $\mathrm{PH}$ analyzed the data. JO wrote the first draft of the manuscript. $\mathrm{CH}$ and $\mathrm{PH}$ wrote sections of the manuscript. All authors contributed to manuscript revision, read, and approved the submitted version.

\section{FUNDING}

This study was funded by the University of Groningen, Groningen, Netherlands.

\section{ACKNOWLEDGMENTS}

The authors thank the children and their parents for participating in this study, Accare Groningen for helping with participant recruitment, and Sanne Kuijper for her statistical assistance. 


\section{REFERENCES}

Ackerman, P. L., Beier, M. E., and Boyle, M. O. (2005). Working memory and intelligence: the same or different constructs? Psychol. Bull. 131, 30-60. doi: 10.1037/0033-2909.131.1.30

Aissen, J. (2003). Differential object marking: iconicity vs. economy. Nat. Lang. Linguist. Theory 21, 435-483. doi: 10.1023/A:1024109008573

Allman, M. J., and DeLeon, I. G. (2009). "No time like the present": time perception in autism," in Causes and Risks for Autism, eds A. C. Giordano and V. A. Lombardi (New York, NY: Nova Science), 65-76.

American Psychiatric Association (2000). The Diagnostic and Statistical Manual of Mental Disorders, 4th Edn. Washington, DC: APA.

American Psychiatric Association (2013). The Diagnostic and Statistical Manual of Mental Disorders, 5th Edn. Washington, DC: APA. doi: 10.1176/appi.books. 9780890425596

Baddeley, A. (2000). The episodic buffer: a new component of working memory? Trends Cogn. Sci. 4, 417-423. doi: 10.1016/S1364-6613(00)0 1538-2

Baron-Cohen, S., Leslie, A., and Frith, U. (1985). Does the autistic child have a "theory of mind"? Cognition 21, 37-46. doi: 10.1016/0010-0277(85)9 0022-8

Blything, L., and Cain, K. (2016). Children's processing and comprehension of complex sentences containing temporal connectives: the influence of memory on the time course of accurate responses. Dev. Psychol. 52, 1517-1529. doi: 10.1037/dev0000201

Blything, L., Davies, R., and Cain, K. (2015). Young children's comprehension of temporal relations in complex sentences: the influence of memory on performance. Child Dev. 86, 1922-1934. doi: 10.1111/cdev.12412

Boucher, J. (2001). “"Lost in a sea of time": time-parsing and autism," in Time and Memory: Issues in Philosophy and Psychology, eds C. Hoerl and T. McCormack (Oxford: Clarendon Press), 111-135.

Boucher, J. (2012). Research review: structural language in autistic spectrum disorder - characteristics and causes. J. Child Psychol. Psychiatry 53, 219-233. doi: 10.1111/j.1469-7610.2011.02508.x

Boucher, J., Pons, F., Lind, S., and Williams, D. (2007). Temporal cognition in children with autistic spectrum disorders: tests of diachronic thinking. J. Autism. Dev. Disord. 37, 1413-1429. doi: 10.1007/s10803-006-0285-9

Boyle, W., Lindell, A. K., and Kidd, E. (2013). Investigating the role of verbal working memory in young children's sentence comprehension. Lang. Learn. 63, 211-242. doi: 10.1111/lang.12003

Buckner, R. L., and Carroll, D. C. (2007). Self-projection and the brain. Trends Cogn. Sci. 11, 49-57. doi: 10.1016/j.tics.2006.11.004

Clark, E. (1971). On the acquisition of the meaning of before and after. Journal of Verbal Learning and Verbal Behavior 10, 266-275. doi: 10.1016/S0022-5371(71) 80054-3

Colle, L., Baron-Cohen, S., Wheelwright, S., and van der Lely, H. K. J. (2008). Narrative discourse in adults with high-functioning autism or asperger syndrome. J. Autism. Dev. Disord. 38, 28-40. doi: 10.1007/s10803-007-0357-5

Colom, R., Abad, F. J., Quiroga, M. A., Shih, P. C., and Flores-Mendoza, C. (2008). Working memory and intelligence are highly related constructs, but why? Intelligence 36, 584-606. doi: 10.1016/j.intell.2008.01.002

Dagenbach, D., and Carr, T. (1994). Inhibitory Processes in Attention, Memory, and Language. San Diego: Academic Press.

De Hoop, H., and Krämer, I. (2006). Children's optimal interpretations of indefinite subjects and objects. Lang. Acquis. 13, 103-123. doi: 10.1207/s15327817la 1302_4

de Ruiter, L., Theakston, A., Brandt, S., and Lieven, E. (2018). Iconicity affects children's comprehension of complex sentences: the role of semantics, clause order, input and individual differences. Cognition 171, 202-224. doi: 10.1016/j. cognition.2017.10.015

De Sonneville, L. (1999). “Amsterdam neuropsychological tasks: a computeraided assessment program," in Cognitive Ergonomics, Clinical Assessment and Computer-Assisted Learning: Computers in Psychology, eds B. P. L. M. den Brinker, P. J. Beek, A. N. Brand, F. J. Maarse, and L. J. M. Mulder (Lisse: Swets \& Zeitlinger), 87-203.

De Vries, M., and Geurts, H. (2012). Cognitive flexibility in ASD; task switching with emotional faces. J. Autism. Dev. Disord. 42, 2558-2568. doi: 10.1007/ s10803-012-1512-1
Diessel, H. (2008). Iconicity of sequence: a corpus-based analysis of the positioning of temporal adverbial clauses in English. Cogn. Linguist. 19, 465-490. doi: 10. 1515/COGL.2008.018

Durrleman, S., Hippolyte, L., Zufferey, S., Iglesias, K., and Hadjikhani, N. (2015). Complex syntax in autism spectrum disorders: a study of relative clauses. Int. J. Lang. Commun. Disord. 50, 260-267. doi: 10.1111/1460-6984. 12130

Ferretti, F., and Cosentino, E. (2013). Time, language and flexibility of the mind: the role of mental time travel in linguistic comprehension and production. Philos. Psychol. 26, 24-46. doi: 10.1080/09515089.2011.625119

Frith, C. D., and Frith, U. (2006). The neural basis of mentalizing. Neuron 50, 531-534. doi: 10.1016/j.neuron.2006.05.001

Gil, S., Chambres, P., Hyvert, C., Fanget, M., and Droit-Volet, S. (2012). Children with autism spectrum disorders have "the working raw material" for time perception. PLoS One 7:e49116. doi: 10.1371/journal.pone.00 49116

Gillberg, C., and Peeters, T. (1995). Autism: Medical and Educational Aspects. London: Whurr.

Heck, R., Thomas, S., and Tabata, L. (2012). Multilevel Modeling of Categorical Outcomes Using IBM SPSS. New York, NY: Routledge.

Hendriks, P. (2014). Asymmetries Between Language Production and Comprehension, Vol. 42. Dordrecht: Springer. doi: 10.1007/978-94-007-6901-4

Hendriks, P., de Hoop, H., Kramer, I., de Swart, H., and Zwarts, J. (2010). Conflicts in Interpretation. London: Equinox Publishing.

Hill, E. (2004). Evaluating the theory of executive dysfunction in autism. Dev. Rev. 24, 189-233. doi: 10.1016/j.dr.2004.01.001

Hollebrandse, B., van Hout, A., and Hendriks, P. (2014). Children's first and second-order false-belief reasoning in a verbal and a low-verbal task. Synthese 191, 321-333. doi: 10.1007/s11229-012-0169-9

Horn, L. R. (1984). "Toward a new taxonomy for pragmatic inference: q-based and R-based implicature," in Meaning, form and use in context: Linguistic applications, ed. D. Schiffrin (Washington, DC: Georgetown University Press), $11-42$.

Jaeger, F. (2008). Categorical data analysis: away from ANOVAs (transformation or not) and towards logit mixed models. J. Mem. Language 59, 434-446. doi: 10.1016/j.jml.2007.11.007

Just, M. A., and Carpenter, P. A. (1992). A capacity theory of comprehension: individual differences in working memory. Psychol. Rev. 99, 122-149. doi: 10. 1037/0033-295X.99.1.122

Kidd, E. (2013). The role of verbal working memory in children's sentence comprehension. Topics Lang. Disord. 33, 208-223. doi: 10.1097/TLD. 0b013e31829d623e

Klein, W. (1994). Time in Language. London: Routledge.

Kort, W., Compaan, E. L., Bleichrodt, N., Resing, W. C. M., Schittekatte, M., Bosmans, M., et al. (2002). WISC-III-NL Handleiding. London: The Psychological Corporation.

Kretschmer-Trendowicz, A., Ellis, J. A., and Altgassen, M. (2016). Effects of episodic future thinking and self-projection on children's prospective memory performance. PLoS One 11:e0158366. doi: 10.1371/journal.pone.015 8366

Kuijper, S., Hartman, C. A., and Hendriks, P. (2015). Who is he? children with ASD and ADHD take the listener into account in their production of ambiguous pronouns. PLoS One 10:e132408. doi: 10.1371/journal.pone.013 2408

Levinson, S. (2000). Presumptive Meanings: The Theory of Generalized Conversational Implicature. Cambridge: MIT Press.

Lewis, R. L., Vasishth, S., and Van Dyke, J. A. (2006). Computational principles of working memory in sentence comprehension. Trends Cogn. Sci. 10, 447-454. doi: 10.1016/j.tics.2006.08.007

Lord, C., Rutter, M., DiLavore, P. C., and Risi, S. (1999). Autism Diagnostic Observation Schedule: Manual. Los Angeles: Western Psychological Services.

Maister, L., and Plaisted-Grant, K. C. (2011). Time perception and its relationship to memory in autism spectrum conditions. Dev. Sci. 14, 1311-1322. doi: 10. 1111/j.1467-7687.2011.01077.x

McCormack, T., and Hanley, M. (2011). Children's reasoning about the temporal order of past and future events. Cogn. Dev. 26, 299-314. doi: 10.1016/j.cogdev. 2011.10.001 
McCormack, T., and Hoerl, C. (1999). Memory and temporal perspective: the role of temporal frameworks in memory development. Dev. Rev. 19, 154-182. doi: 10.1006/drev.1998.0476

McGlone, M. S., and Harding, J. L. (1998). Back (or forward?) to the future: the role of perspective in temporal language comprehension. J. Exp. Psychol. Learn. Mem. Cogn. 24, 1211-1223. doi: 10.1037/0278-7393.24.5.1211

Montgomery, J. W., Magimairaj, B. M., and O'Malley, M. H. (2008). Role of working memory in typically developing children's complex sentence comprehension. J. Psycholinguist. Res. 37, 331-354. doi: 10.1007/s10936-0089077-z

Mullane, J., Corkum, P., Klein, R., and McLaughlin, E. (2009). Interference control in children with and without ADHD: a systematic review of flanker and simon task performance. Child Neuropsychol. 15, 321-342. doi: 10.1080/ 09297040802348028

Münte, T. F., Schiltz, K., and Kutas, M. (1998). When temporal terms belie conceptual order. Nature 395, 71-73. doi: 10.1038/25731

Navon, D. (1978). On a conceptual hierarchy of time, space, and other dimensions. Cognition 6, 223-228. doi: 10.1016/0010-0277(78)90014-8

O'Connell, G., Hsu, C. T., Christakou, A., and Chakrabarti, B. (2018). Thinking about others and the future: neural correlates of perspective taking relate to preferences for delayed rewards. Cogn. Affect. Behav. Neurosci. 18, 35-42. doi: 10.3758/s13415-017-0550-8

Overweg, J., Hartman, C., and Hendriks, P. (2018). Children with autism spectrum disorder show pronoun reversals in interpretation. J. Abnorm. Psychol. 127, 228-238. doi: 10.1037/abn0000338

Owen, A., McMillan, K., Laird, A., and Bullmore, E. (2005). N-back working memory paradigm: a meta-analysis of normative functional neuroimaging studies. Hum. Brain Mapp. 25, 46-59. doi: 10.1002/hbm.20131

Perkins, M. R., Dobbinson, S., Boucher, J., Bol, S., and Bloom, P. (2006). Lexical knowledge and lexical use in autism. J. Autism. Dev. Disord. 36, 795-805. doi: 10.1007/s10803-006-0120-3

Perner, J., and Wimmer, H. (1985). John thinks that mary thinks that..." attribution of second-order beliefs by 5- to 10-year-old children. J. Exp. Child Psychol. 39, 437-471. doi: 10.1016/0022-0965(85)90051-7

Pyykkönen, P., and Järvikivi, J. (2012). Children and situation models of multiple events. Dev. Psychol. 48, 521-529. doi: 10.1037/a0025526

Risi, S., Lord, C., Gotham, K., Corsello, C., Chrysler, C., Szatmari, P., et al. (2006). Combining information from multiple sources in the diagnosis of autism spectrum disorders. J. Am. Acad. Child Adolesc. Psychiatry 45, 1094-1103. doi: 10.1097/01.chi.0000227880.42780.0e

Roberts, J. A., Rice, M. L., and Tager-Flusberg, H. (2004). Tense marking in children with autism. Appl. Psycholinguist. 25, 429-448. doi: 10.1017/ S0142716404001201

Rogers, R., and Monsell, S. (1995). Costs of a predictible switch between simple cognitive tasks. J. Exp. Psychol. Gen. 124, 207-231. doi: 10.1037/0096-3445.124. 2.207
Rutter, M., Le Couteur, A., and Lord, C. (2003). The Autism Diagnostic Interview Revised (ADI-R). Los Angeles: Western Psychological Services.

Schilder, F., and Tenbrink, T. (2001). "Before and after: sentence-internal and -external discourse relations," in Workshop From Sentence Processing to Discourse Interpretation: Crossing the Borders, Utrecht.

Schlichting, L. (2005). Peabody Picture Vocabulary Test-III-NL. Amsterdam: Harcourt Test Publishers.

Schneider, W., Eschmann, A., and Zuccolotto, A. (2002). E-Prime User's Guide. Pittsburgh: Psychology Software Tools.

Scott, W. (1962). Cognitive complexity and cognitive flexibility. Sociometry 25, 405-414. doi: 10.2307/2785779

Stocker, K. (2012). The time machine in our mind. Cogn. Sci. 36, 385-420. doi: 10.1111/j.1551-6709.2011.01225.x

Suddendorf, T., and Corballis, M. C. (2007). The evolution of foresight: what is mental time travel, and is it unique to humans? Behav. Brain Sci. 30, 299-351. doi: 10.1017/S0140525X07001975

Trosborg, A. (1982). Children's comprehension of "before" and "after" reinvestigated. J. Child Lang. 9, 381-402. doi: 10.1017/S030500090000 4773

Wallace, G. L., and Happé, F. (2008). Time perception in autism spectrum disorders. Res. Autism Spectr. Disord. 2, 447-455. doi: 10.1016/j.rasd.2007.09. 005

Welsh, M., and Pennington, B. (1988). Assessing frontal lobe functioning in children: views from developmental psychology. Dev. Neuropsychol. 4, 199-230. doi: 10.1080/87565648809540405

Wimmer, H., and Perner, J. (1983). Beliefs about beliefs: representation and constraining function of wrong beliefs in young children's understanding of deception. Cognition 13, 103-128. doi: 10.1016/0010-0277(83)90004-5

Wing, L. (1996). The Autistic Spectrum: A Guide for Parents and Professionals. London: Constable.

Ye, Z., Kutas, M., St. George, M., Sereno, M. I., Ling, F., and Münte, T. F. (2012). Rearranging the world: neural network supporting the processing of temporal connectives. Neuroimage 59, 3662-3667. doi: 10.1016/j.neuroimage.2011. 11.039

Conflict of Interest Statement: The authors declare that the research was conducted in the absence of any commercial or financial relationships that could be construed as a potential conflict of interest.

Copyright (c) 2018 Overweg, Hartman and Hendriks. This is an open-access article distributed under the terms of the Creative Commons Attribution License (CC BY). The use, distribution or reproduction in other forums is permitted, provided the original author(s) and the copyright owner(s) are credited and that the original publication in this journal is cited, in accordance with accepted academic practice. No use, distribution or reproduction is permitted which does not comply with these terms. 